\title{
An Assessment of Molecular Dynamic Force Fields for Silica for Use in Simulating Laser Damage Mitigation
}

T. F. Soules, G. H. Gilmer, M. J. Matthews, J. S. Stolken, M. D. Feit

November 4, 2010

SPIEL Laser Damage

Boulder, CO, United States

September 26, 2010 through September 29, 2010 
This document was prepared as an account of work sponsored by an agency of the United States government. Neither the United States government nor Lawrence Livermore National Security, LLC, nor any of their employees makes any warranty, expressed or implied, or assumes any legal liability or responsibility for the accuracy, completeness, or usefulness of any information, apparatus, product, or process disclosed, or represents that its use would not infringe privately owned rights. Reference herein to any specific commercial product, process, or service by trade name, trademark, manufacturer, or otherwise does not necessarily constitute or imply its endorsement, recommendation, or favoring by the United States government or Lawrence Livermore National Security, LLC. The views and opinions of authors expressed herein do not necessarily state or reflect those of the United States government or Lawrence Livermore National Security, LLC, and shall not be used for advertising or product endorsement purposes. 


\title{
An Assessment of Molecular Dynamic Force Fields for Silica for Use in Simulating Laser Damage Mitigation
}

\author{
Thomas F. Soules, George H. Gilmer, Manyalibo J. Matthews, James S. Stolken and Michael D. Feit \\ National Ignition Facility and Photon Sciences, Lawrence Livermore National Laboratory \\ 7000 East Avenue, Livermore, California 94550
}

\begin{abstract}
We compare force fields (FF's) that have been used in molecular dynamic (MD) simulations of silica in order to assess their applicability for use in simulating IR-laser damage mitigation. Although pairwise FF's obtained by fitting quantum mechanical calculations such as the BKS and CHIK potentials have been shown to reproduce many of the properties of silica including the stability of silica polymorphs and the densification of the liquid, we show that melting temperatures and fictive temperatures are much too high. Softer empirical force fields give liquid and glass properties at experimental temperatures but may not predict all properties important to laser mitigation experiments.
\end{abstract}

Keywords: Molecular dynamics, silica structure, silica glass properties, laser damage mitigation.

\section{INTRODUCTION}

$\mathrm{CO}_{2}$ laser treatments are a common method of mitigating surface damage sites generated on silica optics by the high fluence laser beams. Depending on the pulse duration and intensity, this process can involve flow and densification and/or evaporation or even ablation. During the $\mathrm{CO}_{2}$ laser pulse the damaged silica region is heated to between 2000 and $5000 \mathrm{~K}$ in $10^{-6}$ to $10^{-12} \mathrm{~s}$. The effects of these laser pulses are being modeled with finite-element tools. However basic properties of silica such as heat capacities, thermal conductivities, thermal expansion and densification, and fictive temperatures under these conditions of very rapid heating and cooling to very high temperatures are often unknown and cannot be determined by conventional experimental methods. Molecular dynamics (MD) has been shown to predict some of the properties of fused silica including atomic structure pair correlation functions, the stability of silica polymorphs and densification. An MD model with realistic force-fields (FF's) may provide these quantities for use in predictive finite-element tools. Also direct MD simulation of the laser mitigation experiment may also be possible and can suggest improvements to the laser mitigation protocol.

The purpose of this paper is to compare simple radial force-fields (FF's) previously used in MD simulations of silica in order to assess their ability to predict the properties of silica that are important in laser damage mitigation by means of a $\mathrm{CO}_{2}$ laser. Four Si-O pairwise FF's are shown in Figure 1 as function of radial distance, $r$, between the atoms along with the analytical expressions for all the distinct pairs: Si-O, O-O and Si-Si. There is a large difference in the Si-O bonding energies from these FF's. The two "strong" fields, $\mathrm{BKS}^{1}$ and $\mathrm{CHIK}{ }^{2}$ were obtained by fitting a Hartree-Fock quantum mechanical calculation and fitting the structure obtained from a density functional Car-Parinello calculation respectively. The two "softer" potentials are empirical with the Takada ${ }^{3}$ force field being the sum of a Morse potential plus Coulomb interactions, and the Soules FF being an ionic Born-Mayer potential with charges scaled to give a reasonable melting temperature ${ }^{4}$. 


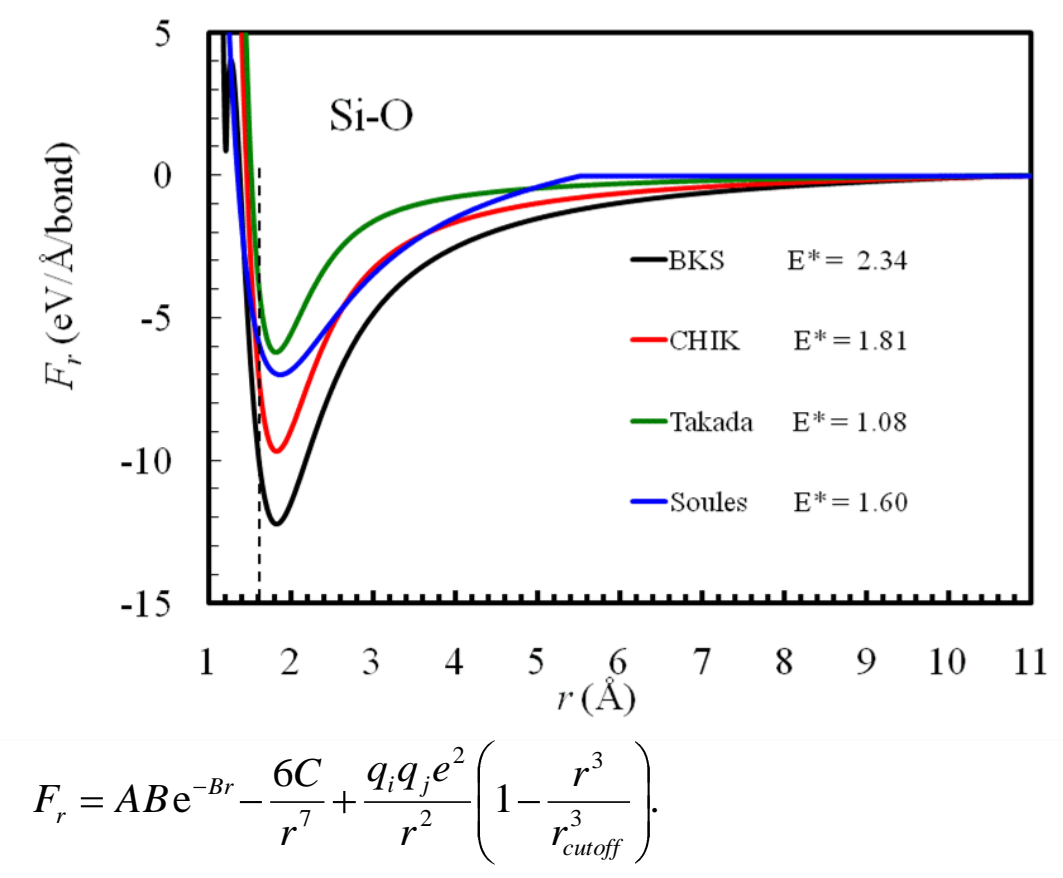

\begin{tabular}{|l|l|l|l|l|l|l|l|l|l|l|l|}
\hline & \multicolumn{2}{l|}{$q_{S i}$} & \multicolumn{1}{l|}{$r_{\text {cutoff }}$ Si-O } & \multicolumn{2}{l|}{ O-O } & \multicolumn{3}{l|}{ Si-Si } \\
& & & $A(\mathrm{eV})$ & $B\left(\AA^{-1}\right)$ & $C\left(\AA^{6}\right)$ & $A(\mathrm{eV})$ & $B\left(\AA^{-1}\right)$ & $C\left(\AA^{6}\right)$ & $A(\mathrm{eV})$ & $B\left(\AA^{-1}\right)$ & $C\left(\AA^{6}\right)$ \\
\hline BKS & 2.4 & 11.0 & 18004 & 4.473 & 133.5 & 1388. & 2.76 & 175 & 0 & 0 & 0 \\
\hline CHIK & 1.910 & 11.0 & 27029 & 5.158 & 148.1 & 659.6 & 2.590 & 26.84 & 3150.1 & 2.852 & 626.8 \\
\hline Soules* & 2.28 & 5.5 & 608.55 & 3.448 & 0 & 466.6 & 3.448 & 0 & 0 & 0 & 0 \\
\hline
\end{tabular}

$F_{r}=-2 A B\left(-\mathrm{e}^{B(C-r)} e^{B(C-r)}+\frac{q_{i} q_{j} e^{2}}{r^{2}}\left(1-\frac{r^{3}}{r_{\text {cutoff }}^{3}}\right)\right.$.

\begin{tabular}{|l|l|l|l|l|l|l|l|l|l|l|l|}
\hline & $q_{S i}$ & $r_{\text {cutoff }}$ & $A(\mathrm{eV})$ & $B\left(\AA^{-1}\right)$ & $C(\AA)$ & $A(\mathrm{eV})$ & $B\left(\AA^{-1}\right)$ & $C(\AA)$ & $A(\mathrm{eV})$ & $B\left(\AA^{-1}\right)$ & $C(\AA)$ \\
\hline Takada & 1.3 & 11.0 & 1.996 & 2.652 & 1.628 & 0.0233 & 1.373 & 3.791 & 0.0077 & 2.045 & 3.760 \\
\hline
\end{tabular}

Figure 1. A graph of the Si-O force-fields (FF's) compared in this work along with the analytical expressions for the FF's and the parameters used. Soules* is from ref. 4, Eq. 9 converted to present units and scaled by 0.65 with the Si-Si short range repulsion set equal to 0 . 


\section{Comparison Results and Discussion}

\subsection{Melting}

In order to compare the melting temperatures from MD simulations with the four FF's, ensembles of 3000 atoms (1000 silicon atoms and 2000 oxygen atoms) were placed at their respective positions in idealized $\beta$-crystobalite in a cube with periodic boundary conditions. The MD run was then carried out at constant pressure ( 1 atmosphere), and the system was heated using the Langevin method. The energy, or in this case, the enthalpy of each system is graphed in Figure 2 versus temperature. The wide range of enthalpies between the four FF's reflects the wide range in Si-O binding energies shown in Figure 1. The small steps seen in the graphs are at the temperatures of homogenous melting, and they reflect the small heats of fusion of $\beta$-crystobalite. The homogenous melting temperature is shown to be dependent on the heating rate as shown in the insert.

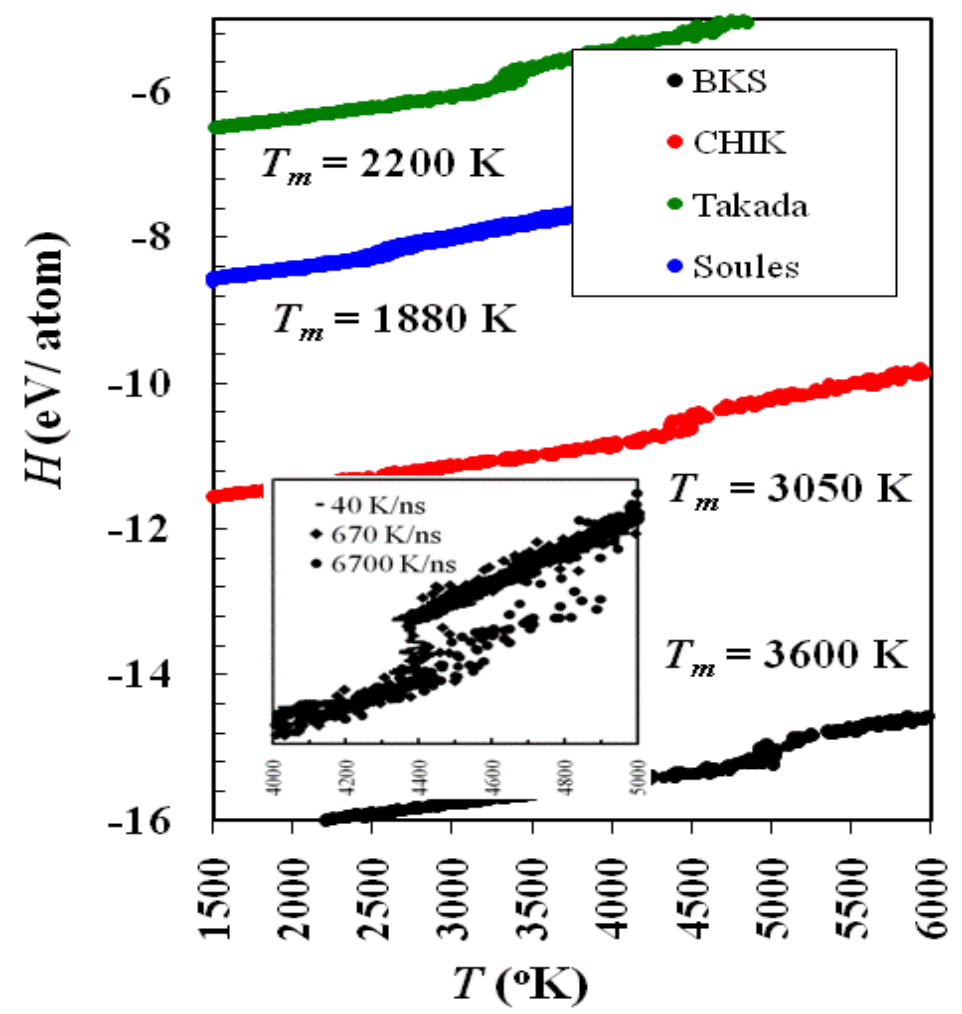

Figure 1 Enthalpy versus temperature during heating of the MD $\beta$-crystobalite ensembles for the four force fields. Kinks in the curves indicate the homogenous melting temperatures when the MD heating is carried out at $4000 \mathrm{~K} / \mathrm{ns}$. Insert shows effect of heating rate on the melting temperature with BKS potential. $T_{m}$ is estimated thermodynamic melting temperatures of the MD ensembles using the methods discussed in the text.

In order to determine the thermodynamic melting points of the different FF's, that is, the temperature at which the crystalline solid and liquid are in equilibrium at a pressure of 1 atmosphere, several methods were used. They involve splitting the MD cell and homogenously melting half of it and then determining the temperature at which the liquid and solid are in equilibrium. The MD methods for determining melting points are described in detail in a paper recently submitted for publication ${ }^{5}$. The temperatures labeled $T_{m}$ in Figure 2 are thermodynamic melting temperatures using these methods. One approach is illustrated in Figure 3. The experimental thermodynamic melting temperature for $\beta$ - 
crystobalite is $\sim 2000 \mathrm{~K}$. As shown in Figures 2 and 3, the BKS and CHIK FF's give unrealistically high values for $T_{m}$.

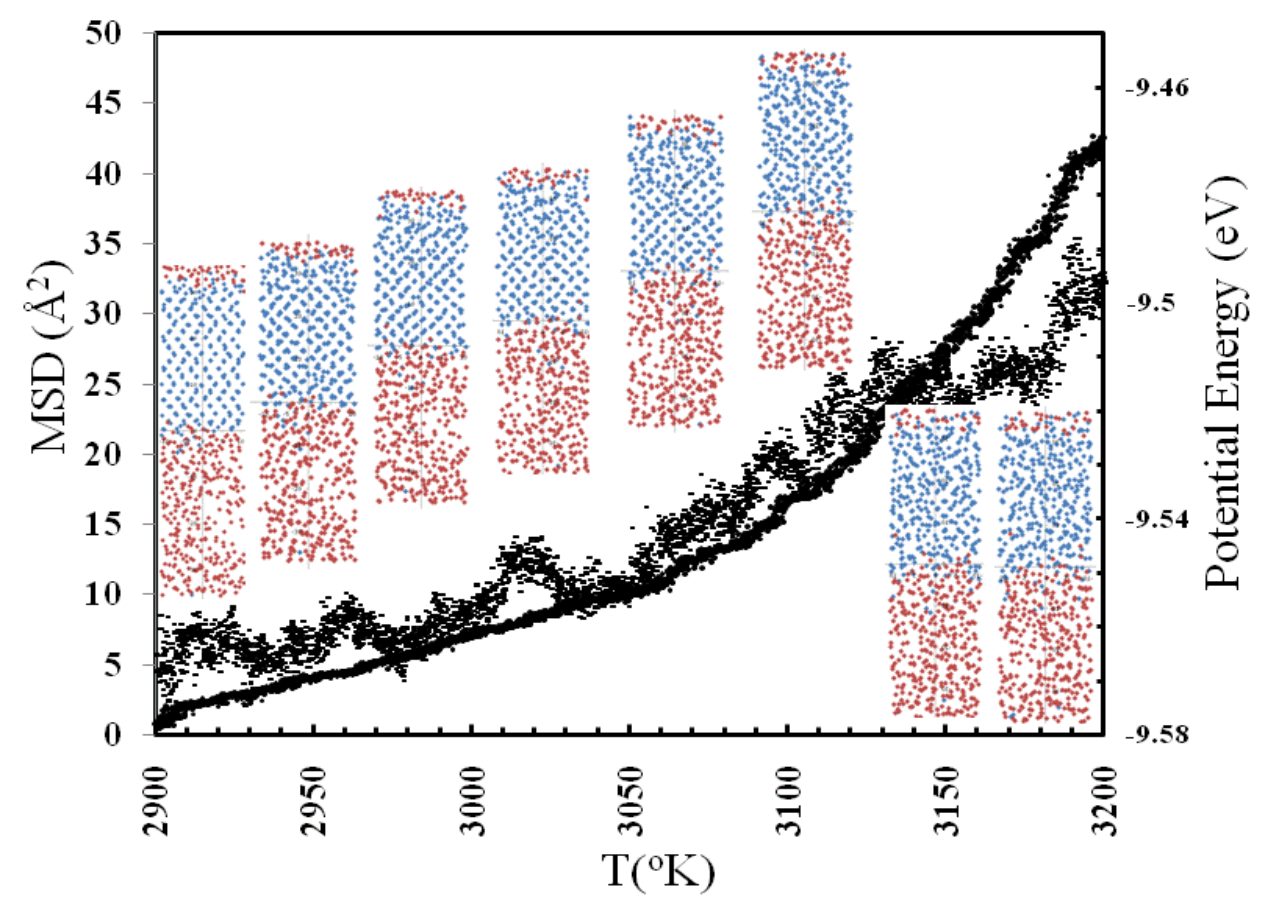

Figure 3 A projection of the silicon atoms in an MD simulation of melting using the CHIK force field and a rectangular prism with periodic boundary conditions. The top half of the cell has the atoms originally in a $\beta$-crystobalite configuration ( $\mathrm{Si}$ atoms as blue diamonds). The bottom half of the cell was homogenously melted (red diamonds). The whole cell is then cooled and heated at the same temperature with a heating rate of $300 \mathrm{~K} / \mathrm{ns}$. The potential energy versus temperature of the system is the data with scatter. The smooth curve is the mean squared displacement (MSD) of the crystal atoms versus temperature. Melting at $\sim 3050 \mathrm{~K}$ is indicated by a change in curvature in the MSD curve, movement of front of disorder into the crystal lattice and a change in slope of the potential energy.

\subsection{Liquid to Glass Transition}

The liquid to glass transition for each FF was assessed by cooling the melted ensembles and determining the fictive temperatures. The fictive temperature is the temperature at which the fluctuating liquid structure is arrested in a local potential energy amorphous minima. A liquid that is cooled very rapidly will have a higher fictive temperature than one that is cooled more slowly, since the latter will continue to sample the potential energy hypersurface to a lower temperature before being arrested on the time scale of the experiment. Figure 4 shows the enthalpy versus temperature during cooling of the MD simulations at a rate of $\sim 1 \mathrm{~K} / \mathrm{ps}$. The liquid to glass transition can be seen as a change in slope of the enthalpy curve, and the fictive temperature is obtained as the intersection of the lines obtained by extrapolating from the liquid and solid curves. 


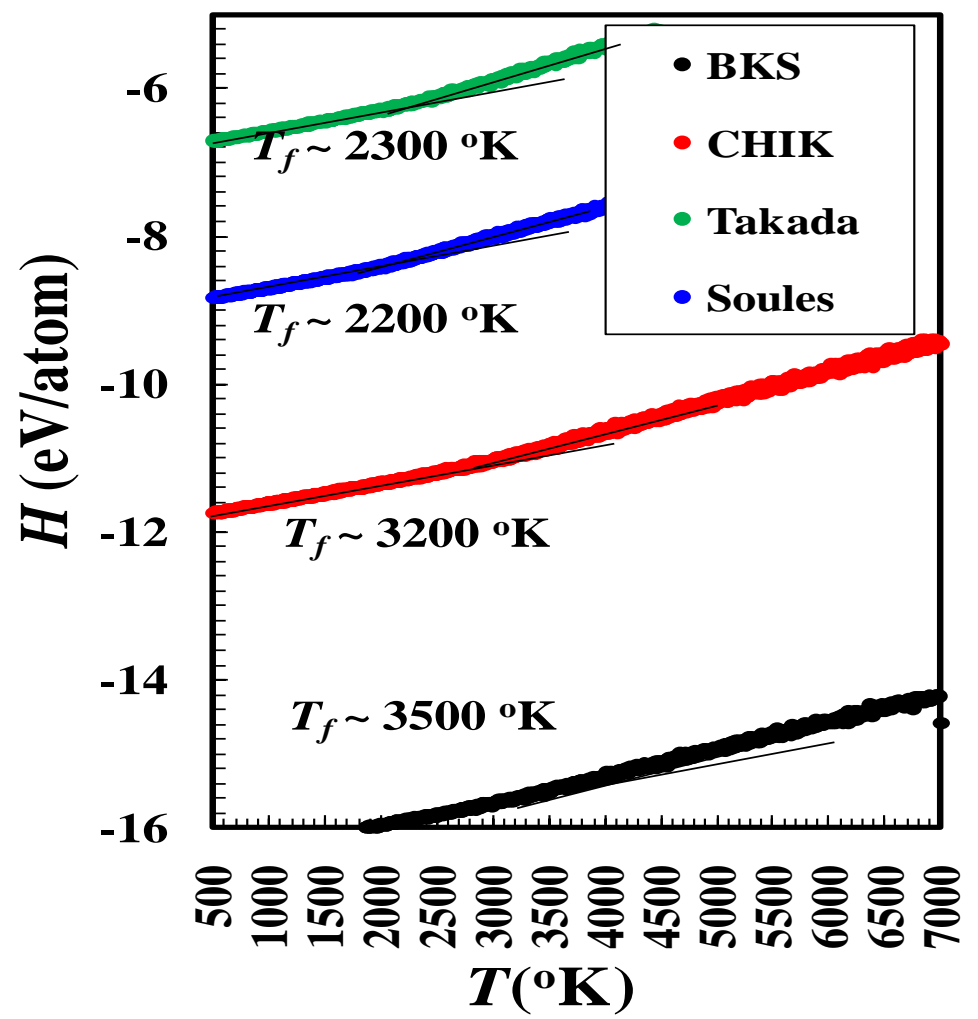

Figure 4 Enthalpy curves for MD simulations during cooling. The cooling rates are 10 million time steps or $10 \mathrm{~ns}$ over the temperature range shown.

Because cooling on MD time scales is always very fast, one expects a high fictive temperature compared to experimental cooling rates although heating and cooling rates close to those in the MD simulations are observed near the laser peak intensity in the $\mathrm{CO}_{2}$ laser mitigation experiments. The fictive temperature can be estimated for our MD cooling using the Tool-Narayanaswamy equation with reasonable parameters.

$$
\begin{aligned}
& T_{f}=T-\int_{T_{o}}^{T} M\left(T^{\prime}, T_{f}, t\right) d T^{\prime} ; \\
& M=\exp \left[-(t / \tau)^{\beta}\right] ; \\
& \tau \sim \tau_{r e f} \exp H / R\left(-1 / T_{r e f}+x / T+(1-x) / T_{f}\right) \quad H / R \sim 73 k K ; \quad x \sim 0.7 ; \quad \beta \sim 1.0 .
\end{aligned}
$$

The result is $\sim 2600 \mathrm{~K}$ for $1 \mathrm{~K} / \mathrm{ps}$. However, there may not be any amorphous minima that correspond to this temperature. The highest fictive temperatures measured to date during our very rapid cooling of silica in laser mitigation experiments are $\sim 2200 \mathrm{~K}$. In any case, assuming that the maximum experimental fictive temperature is $2200-2600 \mathrm{~K}$, the BKS and CHIK FF's give fictive temperatures that are too high.

The heat capacity, or the slope of the enthalpy curve, is shown in Figure 5. At experimental liquid to glass fictive temperatures $(1500-2000 \mathrm{~K})$, there is little change in the heat capacity in going from the solid to the liquid or vice versa implying that there is little heat absorbed in going from one local amorphous minima to another. This has been used to characterize silica as a 'strong' glass. The small change in heat capacity may be due to the random tetrahedral network structure. Topological changes can 
be made by disconnecting and reconnecting different corner sharing tetrahedral with little net change in energy.

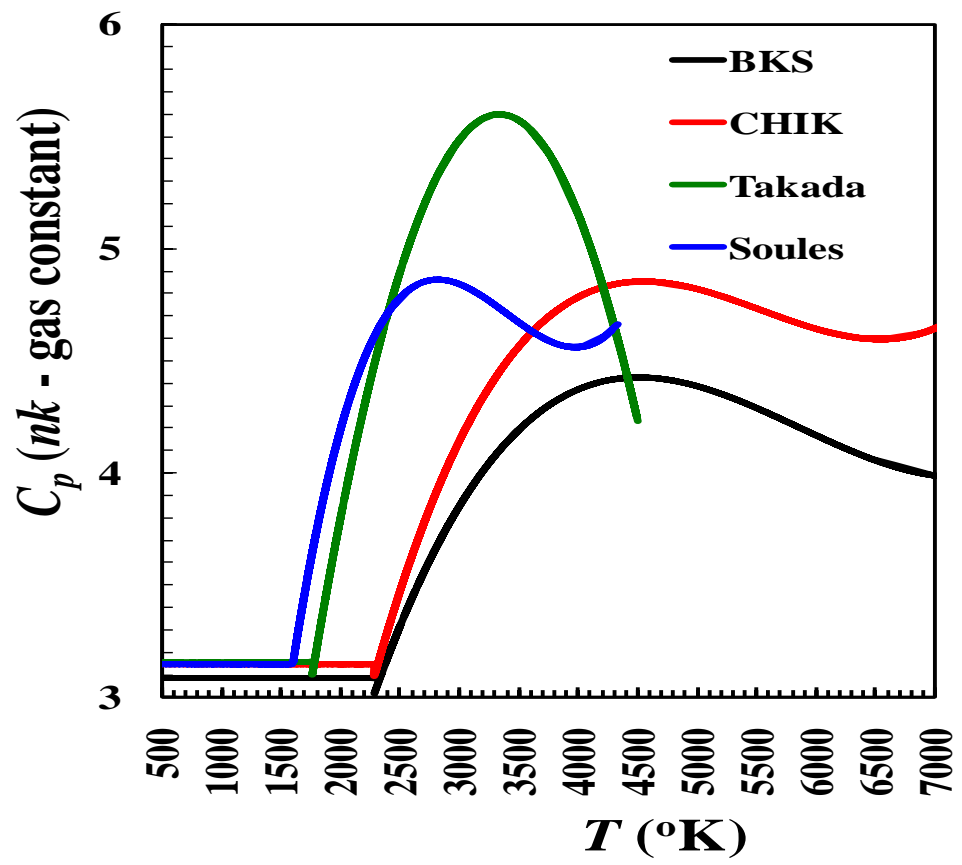

Figure 6. Heat capacity, $C_{p}$, curves obtained by fitting the low temperature enthalpy curves from Figure 5 to a line and then fitting the higher temperature data to a low order polynomial and taking the derivative.

On the other hand, all the FF's predict that the heat capacity will increase significantly by $50-80 \%$ or so at higher temperatures. This is due to the population of higher energy amorphous minima including silicon atoms with 3 or 5 oxygen atoms and singly bonded oxygen atoms. This is characteristic of glasses consisting of randomly close-packed atoms or 'fragile' glasses.

\subsection{Thermal expansion and densification}

Figure 7 shows a comparison of the density of each of the MD runs with each of the FF's as the system is cooled from the high temperature liquid. Experimentally silica undergoes a small densification, $\sim 0.5 \%$ near $1900 \mathrm{~K}^{6}$. In the glass state below the fictive temperature, the solid has a very low volumetric thermal contraction coefficient, $\sim 1.55 \times 10^{-6}$. These properties can be understood from the structure. The atomic structure from all the MD runs show silica tetrahedra connected at corners with a distribution of Si-O-Si bond angles. The connected tetrahedra form rings that are the ribs of an open fused cage-like structure. With increasing temperature the bridging oxygen atoms vibrate normal to the Si-O-Si bond. This motion does not increase the volume, and at some point the structure collapses to a more dense form similar to the low temperature polymorphs. The room temperature density of silica glass is $\sim 2.2 \mathrm{~g} / \mathrm{cm}^{3}$. As can be seen from the graph, the qualitative features of the thermal expansion and densification are reproduced by the MD simulations. However, the BKS and CHIK potentials show densification at a temperature that is much too high. Also, the magnitude of the densification predicted by the BKS and Soules FF's is too large by close to an order of magnitude. The Takada FF on the other hand shows a much larger thermal expansion than observed experimentally and very little or no densification. 


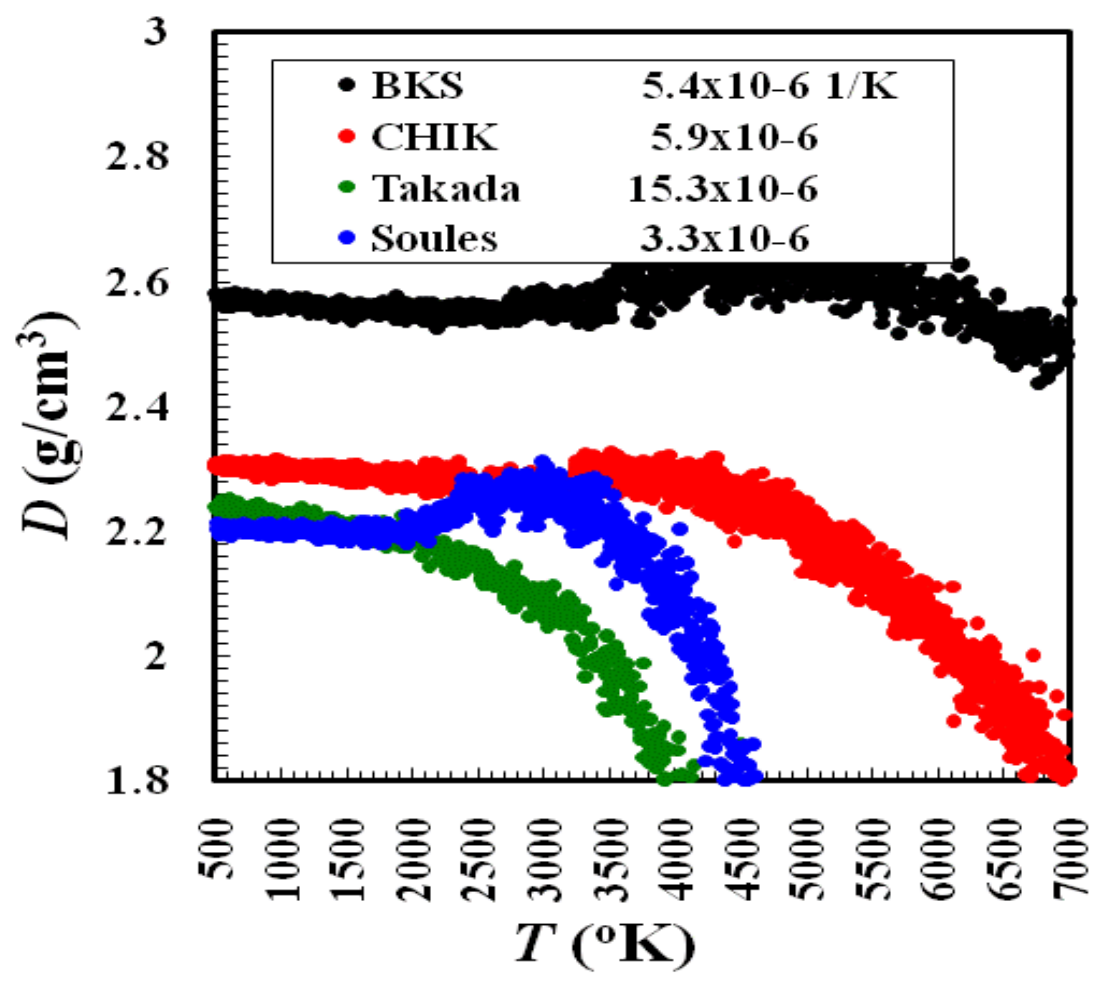

Figure 7. Density during MD cooling for each of the force fields tested. The numbers in the legend are volumetric thermal contraction coefficients per degree $K$ in the solid temperature range $\left(300-1500{ }^{\circ} \mathrm{K}\right)$.

\subsection{Bulk Modulus}

The compressibility or bulk modulus of silica is shown in Table 1 and compared to the results obtained by compressing the room temperature MD ensembles with different FF's. The results for all FF's except Takada which agrees within the error of the measurement and simulation are $~ 30 \%$ higher than the experimental value.

\begin{tabular}{|l|l|}
\hline Model & Bulk modulus (GPa) \\
\hline BKS & $59+/-5$ \\
\hline CHIK & $54+/-4$ \\
\hline Takada & $43+/-3$ \\
\hline Soules & $56+/-5$ \\
\hline Experiment & $\sim 39+/-2$ \\
\hline
\end{tabular}

Table 2. Bulk moduli, $B=(1 / V)(\partial V / \partial T)$, obtained by reducing slightly in steps the volume of the MD simulation box at room temperature. 


\subsection{Self-diffusion coefficients}

Silicon and oxygen atom self-diffusion constants obtained from the slope of the long time mean squared displacements of the atoms are shown in Figure 8. Again, there are significant differences in both the preexponent and activation energies between the strong and weak FF's although all show a close correlation between the diffusion of $\mathrm{Si}$ and $\mathrm{O}$ atoms. Unfortunately, experimental measurements of these diffusion coefficients are difficult and somewhat contradictory. The activation energies for self-diffusion of Si and $\mathrm{O}$ are much less than the activation energy measured for viscosity of silica at these temperatures, namely $\sim 70 \mathrm{kK}$ suggesting that Einstein relation connecting self-diffusion coefficients and viscosity is not valid for these tetrahedrally connected glasses.

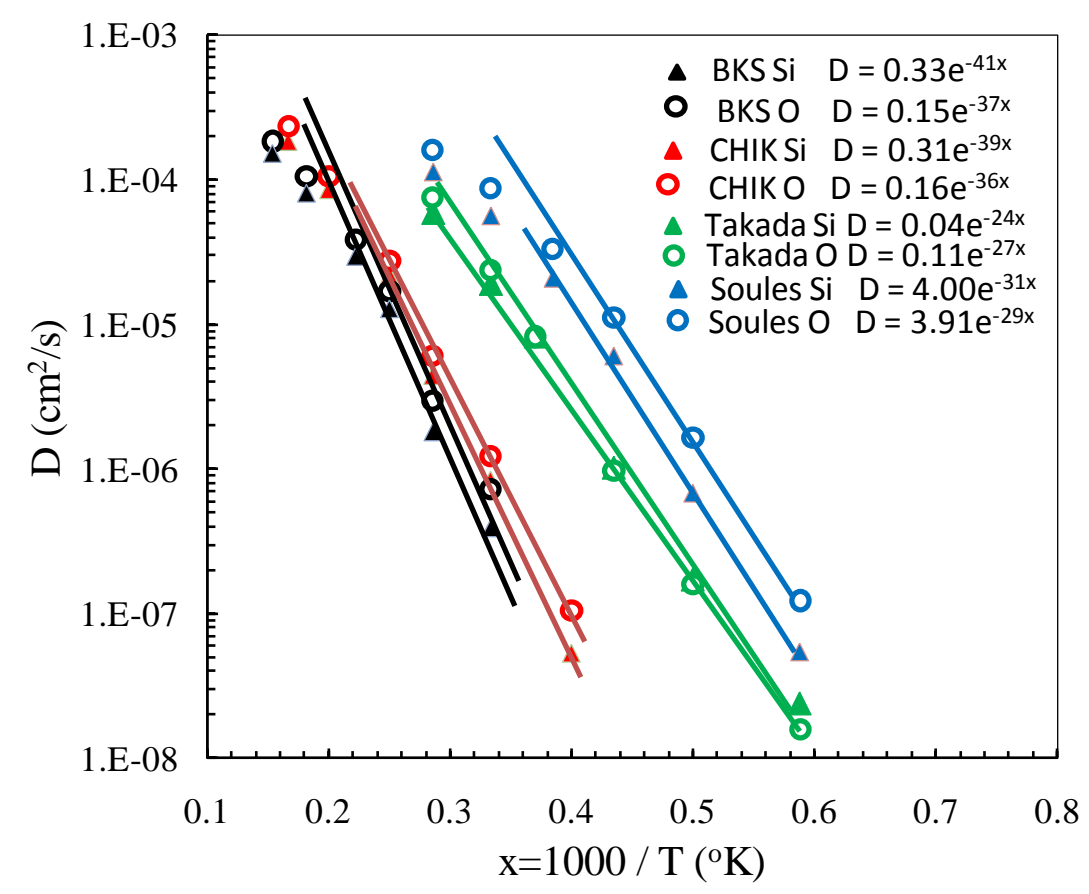

Figure 8. Si and $O$ self-diffusion constants determined from the mean square displacements of atoms evaluated at different temperatures for each of the FF's investigated.

\section{CONCLUSIONS}

While previously used pairwise FF's based on ab-initio quantum mechanical calculations predict the random network structure of silica and give qualitatively several properties of experimental silica, they yield melting points and liquid to glass transitions at temperatures that are too high. Because of the importance of the high temperature properties of silica in laser mitigation experiments and because these properties cannot be easily obtained by conventional methods, the empirical 'soft' FF's are more promising for our simulations. The 'soft' FF's will have to be modified, fine tuned or re-parameterized to better fit the thermal expansion and densification data. This approach along with investigating still other FF's including the ReaxFF's ${ }^{7}{ }^{8}$ are being pursued with the hope that a single set of FF's will reasonably predict all the properties of silica of interest in laser mitigation experiments. 


\footnotetext{
${ }^{1}$ B. W. H. van Beest, G. J. Kramer, and R. A. van Santen, Phys. Rev. Lett. 64, 1955 (1990).

${ }^{2}$ A. Carre, J. Horbach, S. Ispas and W. Kobb, EPL, 82, 17001 (2008).

${ }^{3}$ A. Takada, P. Richet, C. R. A. Catlow and G. D. Price J. Non-crys. Solids, 345-346, 224-229 (2004). E. Demiralp, T. Cagin and W. A. Goddard III, Phys. Rev. Letters, 82, 1708 (1999).

${ }^{4}$ T. F. Soules, J. Non-crys. Solids 123, 48-70 (1990).

${ }^{5}$ T. F. Soules, G. H. Gilmer, M. J. Matthews, James S. Stolken, and Michael D. Feit, submitted for publication to J. Non-crys. Solids (2010).

${ }^{6}$ R. Bruckner, J. Non-crys. Solids 5, 123-175 (1970).

${ }^{7}$ A. C. T. van Duin, S. Daxgupta, F. Lorant, and W. A. Goddard III, J. Phys. Chem. A, 105, 9396-9409 (2001).

${ }^{8}$ A. C. T. van Duin, A. Strachan, S. Stewman, Q. Zhang, X. Xu, and W. A. Goddard III A, 107, 3803-3811 (2003).
} 